\title{
Wellness Behaviors and Body Mass Index Among U.S. Adolescents: A Comparative Study
}

\author{
Timothy Makubuya \\ Department of Educator Preparation and Leadership, University of Missouri- St. Louis, St. Louis, USA \\ Email address: \\ makubuyat@umsl.edu

\section{To cite this article:} \\ Timothy Makubuya. Wellness Behaviors and Body Mass Index Among U.S. Adolescents: A Comparative Study. International Journal of \\ Sports Science and Physical Education. Vol. 3, No. 3, 2018, pp. 32-39. doi: 10.11648/j.ijsspe.20180303.11
}

Received: September 22, 2018; Accepted: October 15, 2018; Published: November 1, 2018

\begin{abstract}
Behaviors such as sedentariness, poor nutritional choices and inadequate sleeping put youth at risk of illnesses. Although health and physical education programs are structured to promote the development of various health and physical skills, they are constantly faced with challenges to their continual existence. As such, issues related to body composition ensue and manifest differently across gender and race/ethnicity. This study aimed at examining the relationships between multiple youth risk behaviors and body composition. In addition, gender and racial/ethnic differences between white and black high school students were examined. Bivariate and multivariate examinations of physical activity, dietary behavior, sleep in relation to Body Mass Index (BMI) percentiles, was followed by a comparison of two data sets of the 2013 YRBS ( $\mathrm{n}=13$, 363) and 2015 YRBS $(n=15,624)$. The results revealed an existence of gender differences in relationships between physical activity, dietary behavior, sleep duration and BMI percentiles with significant associations in male high school students but not their female counterparts in both data sets. There were no racial differences in the strength of these relationships between Black or African American and White or Caucasian high school students. These findings corroborate the need for gender based interventions and further analyses based on non-subjective measures of those health-risk behaviors, in order to fully understand the relationships. In addition, family, school and community based interventions to the physical inactivity, poor nutrition and poor sleep habits are warranted, and should encircle strategies from various stakeholders.
\end{abstract}

Keywords: Physical Activity, Dietary Behavior, Sleep, Body Mass Index’ Race/Ethnicity

\section{Introduction}

A number of schools and school districts across the U.S., often contemplate ways of dealing with budgetary challenges especially during difficult economic times [1]. Health and physical education (PE) programs often become victims of these unforeseeable budgetary shortfalls, leading to their reductions or closures. Physical education should be emphasized as a critical investment in the nation's youth, although monetary decision makers almost often neglect such investment. Even though U.S. Department of Health and Human Services' guidelines for physical activity require meaningful daily physical activity in children and adolescents [2], many children just don't meet the requirements. In addition, many children live in communities and homes, that often lack the capability to provide resources for a healthy living. Often too, the economic gap between rich and poor, exacerbates the situation and hence leading to extreme health disparities between the poor and their wealthy counterparts. Of specific importance though, are the health disparities in physical activity and diet. In addition, to the low socio-economic status (SES) as a major factor [3], African American youth often lack the opportunities to access community recreational resources compared to their white counterparts [4]. Similar patterns and trends are also often evident in schools with higher percentages of diverse students [5].

In fact, it is through health and physical education that many youths obtain meaningful levels of physical activity and other health related skills including nutrition and overall wellbeing. Even though, there is an emphasis on the adoption of a framework that promotes high-quality physical education. In addition to meeting the minimum of 150 minutes of weekly instructions in elementary school and 225 
minutes in middle and high schools, many schools still lag behind [1]. In some cases, the necessary recommendations for daily moderate to vigorous physical activity for children and adolescents are not being met.

Although from the most recent data, rates of overweight and obesity in adolescents are levelling off, the fact is that, those rates are still exorbitant. Conversations around body composition should often require an examination of school and out of school nutrition practices, with their unavoidable interrelationship with physical inactivity. Conversely, instead of only focusing on nutrition and physical inactivity, there is also need to study the role of other factors outside schools, such as insufficient sleep and higher risk of obesity among children. This aspect of health and wellness could better be addressed through family structures. Available evidence suggests that, there is an expeditiously increasing literature on chronic partial sleep [6] from both epidemiological and clinical studies indicating its link to obesity risk and weight gain [7]. An analysis of national YRBS data examined the association between self-reported obesity and self-reported sleep duration, and found results to exhibit gender differences, with girls having significant association compared to their male counterparts [8], although the recommendations suggested caution on abrupt sleep adjustment practices, citing the need for further studies.

Physical activity, nutrition and sleep habits have received heightened attention in studying relationships with body composition among all individuals. Previous investigations [9] conducted to establish the relationship between physical activity and overweight children in Taiwan suggested that the emphasis should be placed on extrinsic factors and how they impact overweight and obesity in children. These influences are normally within school, community and family control. A two-year longitudinal study reported both diet-related and physical activity predictors of obesity in young children between 6 and 9 years old [10]. About 48\% of those respondents were Latinos and their findings suggested that there was an interrelatedness between the factors of diet, physical activity and obesity. Elsewhere, food patterns, physical activity, sleep, television viewing, and longer sleep hours were associated with consumption of fast food, the lack of vegetables and fruit-rich diet among Portuguese children [11]. Television viewing often increases the sit-down time, which is fountainhead to sedentariness and sitting disease syndrome.

Adolescents greatly benefit from physical activity and physical education classes, and this is particularly under the guidance of a teacher. Some would also argue that engagement in other extracurricular activities would provide similar benefits. A study to compare the outcomes from two groups with one engaging in physical education and the other in Junior Reserve Officer Training Corps (JROTC), a commonly used alternative for Physical Education (PE) time [12] revealed significant differences in instruction time, content and context, with students engaging in more moderate to vigorous physical activity during $\mathrm{PE}$ than JROTC. Therefore, such revelations should spearhead advocacy efforts for programs that meet and exceed the daily recommendation for the right intensity and duration of physical activity. The purpose of this study was to examine the gender and racial/ethnic related association between physical activity, diet, and sleep with body mass index percentiles and then compare the results of two crosssectional data sets. In addition, this paper conceptualizes the Whole School, Whole Community and Whole Child approach, that addresses school, and community institutions through connections that require family engagement in the exploration of potential school and community resources and interventions aimed at addressing the health and wellness behaviors that lead to overweight and obesity.

\section{Materials and Methods}

\subsection{Participants and Setting}

Data from participant responses on the 2013 and 2015 youth risk behavior survey (YRBS) were used to identify and examine health risk behavior items about physical activity, food and sleep for 9th to 12th grade high school students. Both national samples for the 2013 and 2015 YRBS were determined using a three-stage cluster sample design by the U.S. Center's for Disease Control and Prevention (CDC). Participants were from selected private and public schools that were in the representative pool.

After all multi-level eliminations of missing cases and removing outliers at three standard deviations from the mean, 13,363 and 15,624 participant questionnaires were used for both 2013 and 2015 analyses respectively. These participants identified as both male and females from the 9th and 12th grade classes that had a varying racial/ethnic make-up.

\subsection{Data Collection}

YRBS data were collected nationally from sampled schools following similar procedures in 2013 and 2015 [13, 14]. The collection of data from respondents required passive consent that was gained through permission slips that were sent home from schools, requiring adult consent. National YRBS data were collected by CDC and its vendor trained collectors who presented students with computer readable answer sheets with the survey. Once data were collected through responses to the different questions on survey, the $\mathrm{CDC}$ and its contractor, ICF Macro, Inc., carried out the preparation and analysis of the initial data [13, 14]. For purposes of this study, the CDC availed the data, and with the approval of the University of Missouri-St. Louis Institution Review Board, for further analysis.

\subsection{Methodology}

Relationship of BMI percentiles with physical activity, dietary behavior and sleep was examined, while controlling for gender and race/ethnicity. Survey responses for physical activity, dietary behavior and sleep elements were scored based on the particular response options per question. Responses on the survey ranged from $\mathrm{A}$ to $\mathrm{H}$ whereby, $\mathrm{A}$ 
represented 0 and $\mathrm{H}$ represented 7 points respectively.

The risk behaviors were then categorized based on an aggregate score of the values for each question ranging from 0 to 7 .

Based on the nature of the health risk behavior question, responses were then assigned a positive or negative numerical value 1 to 7 or -1 to -7 for categories of health risk behavior depending on whether they are positive or negative behaviors. Behaviors such as drinking soda (including non- diet soda), watching TV, and playing video game were assigned a negative numerical value whereas behaviors such as daily participation in 60 minute of physical activity were assigned a positive value. In this study, the question on "how many times did you eat potatoes" was eliminated from the dietary behaviors variable computation from both 2013 and 2015 data. Physical activity was grouped into three categories (Nearly Always/ Often, Occasional and Little/No Physical Activity); dietary behavior into three categories (Frequent Consumption, Moderate Consumption, Little /No Consumption); and hours of sleep into three categories (Recommended, Close to Recommended and Far Less than Recommended). Higher physical activity scores implied nearly always or often physically active participation, whereas frequent consumption implied higher scores for the dietary score. Longer sleep durations were the equivalent of gaining the recommended sleep duration and vice versa.

Since YRBS questions on physical activity were mainly reported based on the frequency of their occurrence. This study categorized these responses based on the SHAPE America Physical Activity guidelines for adolescents that require daily participation in physical activity. Questions on dietary behaviors were mainly reported based on the frequency of their occurrence. The study categorized these responses based on the USDA Dietary Guidelines for Americans (2015-2020). These guidelines support the recommendations that require frequent/daily consumption of nutritious foods across all food groups by children and adolescents. The National Institute of Health (NIH) recommendations for adolescent health are in line with the CDC recommendations for healthy sleep. The National Sleep Foundations has recommendations for teenagers to obtain 810 hours of sleep daily [15]. Body Mass Index percentiles, an index for body composition was computed from the selfreported weight and height by the respondents. Computations of BMI percentiles were performed by the CDC using the growth chart measures [14].

Table 1. Categories for Health and Wellness Risk Behaviors.

\begin{tabular}{lll}
\hline Physical Activity & Dietary Behaviors & Sleep Duration \\
\hline Nearly Always (5- 7 days) & Frequent Consumption (5-7 days) & Recommended (8-10+ hours) \\
Occasional (3-4 days) & Moderate Consumption (3-4 days) & Close to Recommended $(6<8$ hours $)$ \\
Little ( $<3$ days $)$ & Little/No Consumption ( $<3$ days $)$ & Less than Recommended $(<6$ hours $)$ \\
\hline
\end{tabular}

\subsection{Statistical Analysis}

Bivariate and multivariate measures were used to determine the relationship between the three independent variables of physical activity, dietary behaviors and sleep and the dependent variable of body mass index. Due to the continuous nature of the variables, Spearman Rho correlation was applied to establish the relationship between each independent variable with the dependent variable. Correlation coefficient, $r$ values range from -1 to +1 . In addition, the relationship between these independent variables and the dependent variable was examined with multiple linear regression using Statistical Program for Social Sciences (SPSS 23).

Multiple linear regression, an extension of simple linear regression, was used for this study based on the general equation:

$$
\mathrm{Y}=\beta_{0}+\beta_{1} \mathrm{X}_{1}+\beta_{2} \mathrm{X}_{2}+\beta_{3} \mathrm{X}_{3}+\ldots \ldots \ldots \beta_{\mathrm{n}} \mathrm{X}_{\mathrm{n}}+\text { error }
$$

Under this analysis, the assumption for an error value of 0 is necessary. Therefore, the estimate value $\mathrm{E}$, of the dependent variable Y (BMI percentile), for this study was given by the mathematical equation:

$$
\mathrm{E}(\mathrm{Y})=\beta_{0}+\beta_{\mathrm{pa}} \mathrm{PA}+\beta_{\mathrm{db}} \mathrm{DB}+\beta_{\mathrm{s}} \mathrm{S}+\beta \mathrm{g}
$$

Where $\mathrm{Y}$ is the predicted, expected or estimated dependent variable of BMI percentile, pa is Physical Activity, db is Dietary Behavior, and $\mathrm{s}$ is Sleep, all independent predictors or variables whereas $\beta_{0}$ is the constant, $\beta_{\mathrm{pa}}, \beta_{\mathrm{db}}$ and $\beta_{\mathrm{s}}$, and $\beta_{\mathrm{g}}$ are regression coefficients for physical activity, dietary behavior, sleep and gender respectively. A model that accounted for a higher variance in the prediction was more reliable, and is more acceptable.

For both males and females, emphasis was placed on the two groups of Race/Ethnicity (White and African American/Black) that were used as categorical independent variables in this study. A multiple regression equation that related the outcome or dependent variable of Y (BMI Percentile) to each independent variable of Physical Activity (PA), Dietary Behavior (DB) and Sleep (S) and their product, while checking for interactions and confounding for gender and race/ethnicity was estimated. The interactions were expressed as:

Physical Activity (PA) and Race/Ethnicity (RE) = PA by RE Interaction Variable (PA. RE)

Dietary Behavior (DB) and Race/Ethnicity $(\mathrm{RE})=\mathrm{DB}$ by RE Interaction (DB. RE)

Sleep (S) and Race/Ethnicity (RE) $=\mathrm{S}$ by RE Interaction (S. RE). 


\section{Results}

Table 2. BMI Associations with Health Risk Behaviors.

\begin{tabular}{llllll}
\hline \multirow{2}{*}{ Category } & & $\mathbf{2 0 1 3}$ & & $\mathbf{2 0 1 5}$ & \\
\cline { 3 - 6 } & & $\boldsymbol{r}$ & $\boldsymbol{p}$-value & $\boldsymbol{r}$ & $\boldsymbol{p}$-value \\
\hline \multirow{2}{*}{ Physical } & All & -.31 & .001 & -.28 & .020 \\
Activity & Boys & $.74^{* *}$ & .000 & $-.56^{* *}$ & .000 \\
& Girls & .01 & .958 & .03 & .829 \\
Dietary & All & -.22 & .015 & -.10 & .270 \\
Behavior & Boys & $-.32^{* *}$ & .014 & $-.45^{* *}$ & .001 \\
& Girls & -.15 & .167 & .17 & .200 \\
\multirow{2}{*}{ Sleep } & All & -.31 & .001 & -.09 & .334 \\
& Boys & $-.47^{* *}$ & .000 & -.21 & .107 \\
& Girls & -.19 & .148 & -.04 & .778 \\
\hline
\end{tabular}

$* * \mathrm{P}$ value $<.005$

\subsection{Physical Activity and BMI Percentiles}

\section{YRBS}

Bivariate analysis using Spearman's Rho yielded a significant, negative correlation between BMI percentile and physical activity score among high school students $(\mathrm{r}=-.31$, $\mathrm{p}=.001)$. This indicates that the higher levels of physical activity among high school students, the lower the BMI percentiles. Spearman's Rho correlation between PA score and BMI percentile yielded a significant, negative correlation among high school boys from the 2013 data $(r=-.74$, $p$ $=.000)$ and a weak and non-significant correlation among high school girls $(\mathrm{r}=.01, \mathrm{p}=.958)$.

\section{YRBS}

The correlations between BMI percentiles and physical activity also yielded a significant, negative correlations among high school students $(\mathrm{r}=-.28, \mathrm{p}=.020)$. Among high school boys, the correlations were significant and negative $(r$ $=-.56, \mathrm{p}=.000$ ) and a non-significant correlation among high school girls $(r=.03, p=.829)$. Consequently, higher levels of physical activity were found to be associated with significantly lower BMI percentiles in high school boys and not among high school girls.

\subsection{Dietary Behaviors and BMI Percentiles}

\section{YRBS}

Spearman's Rho yielded a weak negative correlation between dietary behavior score and BMI percentiles from the 2013 data $(\mathrm{r}=-.22, \mathrm{p}=.015)$. Spearman's Rho yielded a weak negative correlation between $\mathrm{DB}$ score and BMI percentiles among high school boys $(\mathrm{r}=-.32, \mathrm{p}=.014)$. Spearman's Rho also yielded a weak negative correlation between dietary behavior score and BMI percentiles among high school girls $(\mathrm{r}=-.15, \mathrm{p}=.167)$. However, this correlation was non-significant at the set $\mathrm{p}$ value. These results suggest that, higher dietary behavior scores are associated with lower BMI Percentiles in high school boys but not in high school girls.

\section{YRBS}

Bivariate correlations yielded indirect associations between dietary behaviors and BMI percentiles $(r=-.10$, $p$ $=.270$ ) among all high school students. However, the correlations were different between males $(\mathrm{r}=-.45, \mathrm{p}=.001)$ and females $(\mathrm{r}=.17, \mathrm{p}=.200)$. The associations between dietary behavior and BMI percentiles among male high school students were both significant, negative and indirect in nature. Therefore, this suggests that higher dietary behavior scores are associated with lower BMI percentiles in high school boys but not in girls.

\subsection{Sleep and BMI Percentiles}

\section{YRBS}

Spearman's Rho yielded a negative and weak correlation, but yet significant at the set $\mathrm{p}$ value for the 2013 data $(\mathrm{r}=$ $.31, \mathrm{p}=.001)$. For boys, longer sleep durations were associated with lower BMI percentiles $(\mathrm{r}=-.47, \mathrm{p}=.000)$. In girls, the results were not significant $(r=-.19, \mathrm{p}=.148)$. The differences in correlations between boys and girls were tested for significance of the difference using a $\mathrm{z}$-test $(\mathrm{p}=.000)$ and this result was significant at $p<.05$. The results implied that the longer the sleep duration by high school boys, the lower their BMI percentiles, unlike in their female counterparts.

\section{YRBS}

Analyses from the 2015 data, yielded non-significant correlation $(r=-.09, p=.334)$ among high school students. The 2015 data also yielded negative but non-significant correlations between sleep duration and BMI percentiles $(\mathrm{r}=$ -. $21, \mathrm{p}=.107)$ among males. In addition, there were nonsignificant negative correlations in females $(r=-.04, p$ $=.778$ ). This analysis didn't confirm the significance of the relationship between sleep duration and BMI percentiles among high school students overall or between boys and girls based on the 2015 data. The results of the 2013 and 2015 analyses for sleep duration differ in terms of significance.

\subsection{Race/Ethnicity}

To examine whether the relationship between the three health risk behaviors and BMI Percentiles was stronger for White or Caucasian than Black or African American high school students depended upon the values as reflected by the regression coefficients.

$\mathrm{E}(\mathrm{Y})=\beta_{\mathrm{o}}+\beta_{\mathrm{pa}} \mathrm{PA}+\beta_{\mathrm{db}} \mathrm{DB}+\beta_{\mathrm{s}} \mathrm{S}+\beta_{\mathrm{paxre}} \mathrm{PA} \cdot \mathrm{RE}+\beta_{\mathrm{dbxre}} \mathrm{DB} \cdot \mathrm{RE}+\beta_{\mathrm{sxre}} \mathrm{S} . \mathrm{RE}$

For both 2013 and 2015, several assumptions for using linear regression that were met. The assumption of linearity was met through examining scatter plots, normality of the distribution was tested using the Kolmogorov-Smirnov test, independence of errors was met through the exclusion of outliers within \pm 3 standard deviations, and homoscedasticity was met through the examination of the residual and scatter plots. To test the strength of the relationship between Caucasian or White and African American or Black, multiple linear regressions was used initially, and an interaction effect included depending on the whether the interaction was significant for the independent variables as selected by the regression model. Some variables such as gender and sleep showed initial significance at $p<.05$, for both 2013 and 2015 data, however, the final model results didn't provide 
meaningful estimation models. Other variables were excluded from the models, due to their lack of significance. There was inconclusive evidence from this study to suggest the strength differentials in the relationships between youth risk behaviors and BMI percentiles among black and white high school students in $2013\left(\beta_{\text {re }}=-4.421, p<.05\right)$ and in $2015\left(\beta_{\mathrm{re}}=-4.543, p<.05\right)$ based on the regression coefficients.

\section{Discussion}

This study precedes any other in comparing two YRBS data sets in examining associations between health risk behaviors of physical activity, dietary behavior and sleep with BMI Percentile. The health- risk behaviors used as variables in this study continue to receive heightened attention in ways that they relate to overall health among individuals of this group, irrespective of gender or race. The results of this study encourage further examinations using non-subjective measures of physical activity, dietary behavior and sleep. Considerations for additional questions or items that address specific behaviors, are necessary. From the results of the analyses, there are specific implications for each health-risk behavior and body mass index, as they relate to adolescent health. Gender differences in physical activity have been documented in previous YRBS research. These were evident in physical activity and weight status associations among high school students surveyed with the 1999 YRBS. A previous study [12] utilized a unique approach that characterized physical activity in different dichotomous variables. This necessitated the examination of the link with weight status based on the CDC weight status categories. The results in that study highlight a similar pattern revealed in the current study [16].

The current study confirms that the gender differences in associations between physical activity and BMI percentiles exist, and this is well supported by a 2016 report by the World Health Organization (WHO) that purported that female adolescents aged 11-17 years of age, were less active than boys in 2010. Whether the chronically low level of physical activity affects the female adolescent's other health outcomes and behaviors, that can indirectly impact body composition, is not revealed by this study. Physical inactivity among girls have been linked to serious illnesses that warrant attention. Previously, a decline in physical activity participation with aging has been established among girls [17]. A review of literature also suggests that the decline in physical activity over the past couple of decades is negatively impacting life expectancy [18]. Across the board strategies that address physical attribute concerns among girls, and peer-pressure related obstacles to physical activity should be encouraged as well as monitored, in ways that reduce the sedentary behavior. Of recent, multi- layered approach to other health issues such as metabolic syndrome is receiving heightened attention [19].

With the persistence of the overweight and obesity problem, especially in children and adolescents, schools and communities at large are continuously implementing wellness policies that are intended to reduce childhood obesity, although they are often challenged by budgetary cuts [1]. To this end, behaviors engaged in away from school, remain a challenge to boys and girls alike. From the results of this study, nutrition and weight status are remarkably important aspects of our daily lives. The Healthy People 2020 objectives address the prevention of excess weight gain in children and adolescents aged 2 to 19 years. This study uncovered gender differences in dietary behaviors similar to those indicated in a previous study of 878 adolescents aged between 11 and 15 years. These were studied for diet, physical activity and sedentary behaviors as risk factors for overweight [20]. There were noticeable differences between these racially diverse girls and boys in terms of percentage calories consumed, as total calories expended per day, and fiber grams per day. Though this revelation seems interesting in terms of comparisons, the studies did not use a similar approach to defining dietary behaviors, since the two studies differ in design and setting. Conversely, the participants in the current study were slightly older based on the grade levels of participants in YRBS. A randomized control approach was used, compared to the self-reported dietary behaviors that are common with the YRBS [20]. In addition, the sample size was also very small compared to the national sample of YRBS. Addressing dietary related problems requires a wider range of approaches that address the need for a cultural shift in nutrition, particularly for foods served in schools and community settings alike. Interestingly also, is the fact that plant based foods may not be widely appealing to many adolescents. Yet, their inclusion in daily meals is fundamental as indicated in the dietary guidelines [21]. Therefore, community and school health educators should continue to emphasize the advantages of plant based nutrients, low fat dairy products and lean and non- processed meats in maintaining health body weights among adolescents, especially since they are nearing a more critical entry stage to adulthood.

The current study also revealed noticeable gender differences in associations between sleep durations and BMI percentiles. There were significant indirect relationships between sleep duration and BMI percentiles among boys and non-significant indirect relationships in their female counterparts. The results are equivocal with an implication that further research on sleep duration within different weight status categories is indispensable. A previous study highlights that the vagueness may also originate from the fact that there is merely a single question on the YRBS on sleep [22]. In addition, relationships within different weight status categories could possibly reveal valuable information to address sleep deficits especially in categories that don't meet the recommended durations. In addition, the incidence of early onset of metabolic syndrome, overweight and obesity, and a cluster of related abnormalities are becoming increasingly recognizable in adolescents [22, 23]. Other studies that found associations between children's weight status and sleep have addressed other aspects of sleep, such 
as sleep quality. Relationships between sleeping less than 8 hours with higher BMI in adolescents regardless of gender have been previously established [23]. Significant associations between sleep duration and BMI, have been detected in females, although ethnic differences in sleep durations didn't exacerbate the BMI disparities [24]. The present study revealed and affirmed that longer sleep durations in boys were associated with lower BMI percentiles unlike in girls. In a previous study, among the factors identified, were further parental sensitization as well as addition of more YRBS items addressing other sleep related aspects [22]. Interventions aimed at addressing low levels of physical activity, poor nutrition and inadequate sleep can be implemented through communities, school and at home.

Community-based overweight and obesity interventions supported by existing infrastructure and resources are able to address these challenges in diverse communities. Community interventions that include reforms to policies in schools and communities, as well as school to home programs have been modestly successful among U.S. youth aged 2- 19 years of age [25]. These community interventions are based on a multiple component approach and guided by the socio-ecological model in addressing a complex overweight and obesity epidemic, which has numerous causes $[25,26]$. However, few interventions have used the Community Based Participatory Research (CBPR) that would otherwise engage various stakeholders [25]. There is evidence to the dangers of isolating key elements of a community wide collective approach to overweight and obesity prevention. Although environmental and policy interventions have been successful in increasing the levels of PA in schools, among male students, the failure to include health instruction, a key contributor to poor eating habits leads to ineffectiveness in these interventions, especially if school nutrition is ignored [27].

One of the challenges to schools, especially those in areas of low socio-economic status (SES), is the ability to maintain valuable co-curricular programs, especially those that would otherwise provide youth with the necessary amount of physical activity, and probably other aspects of health and wellness education that greatly impacts their weight status. Girls are particularly concerned of weight status issues, and often arising from peer pressure. A gender specific school overweight and obesity interventions for girls in low SES in New Zealand revealed the possibility to reverse declining levels of PA with the possibility of preventing unhealthy weight gain [28]. In the U.S., previous school-based interventions have been designed to include and emphasize moderate to vigorous physical activity [29]. What is interesting however is that this intervention included both weekday and weekend opportunities for prescribed physical activity and hence extending the intervention from school to homes [29]. In Europe, interventions have combined both PA and nutrition [30]. Overall, reviews of all interventions advocate for a combination of school-based physical activity and nutrition with family involvement [31].

Many family-based obesity prevention interventions have failed to target media and sleep. In addition, the available literature mostly targets family interventions in non-diverse family backgrounds [31]. There is need to address overweight and obesity from a multi-level and multi-layered approach involving various stakeholders as factors leading to obesity are interconnected [32]. School and home collaboration is necessary in obesity prevention intervention, especially as necessary for adult and parental support in attaining the recommended levels of PA and good nutrition [32]. Interventions that are longer and encompass aspects of PA with family involvement, seem to benefit young and female participants, and don't necessarily aim at environmental policy changes [29]. In addition, there is need for obesity prevention interventions that address sleep quality and behaviors in youth in addition to media usage as controlled by parents of very young and older children alike $[33,34]$.

\section{Conclusions}

This paper indicates the complexity of numerous health risk behaviors, and their impact on weight status in adolescents. Although the results from the correlation analysis, indicated statistical significance, especially in males, cautionary interpretation is indispensable due to the existence of a number of factors that are not addressed by the survey. This study didn't reveal any differences in the strengths of the associations between the three health-risk behaviors and BMI percentiles in black and white high school students, suggesting that it is possible that the effects of physical inactivity, poor dietary choices, and lack of enough sleep affects U.S. high school students in similar ways. In addition, the factors exacerbating overweight and obesity are numerous and complex, thereby requiring a multi-level approach with various stakeholders. Due to the subjective nature of the YRBS, objective and longitudinal data collection tools are encouraged to further similar examinations on these associations.

\section{Acknowledgements}

This study was partially supported by a dissertation grant from the College of Education at University of Missouri- St. Louis.

\section{Author Contributions}

TM solely contributed to this manuscript.

\section{Conflicts of Interest}

The authors declare that they have no competing interests.

\section{References}

[1] Society of Health and Physical Educators (SHAPE America). (2016). Shape of the Nation Report, 2016. Retrieved from www.shapeamerica.org/shapeofthenation. 
[2] Strong, W. B., Malina, R. M., Blimkie, C. J., Daniels, S. R., Dishman, R. K., Gutin, B., Hergenroeder, A. C., \& ... Trudeau, F. (2005). Evidence based physical activity for school-age youth. Journal of Pediatrics, 146(6), 732-737. https://doi.org/10.1016/j.jpeds.2005.01.055.

[3] Eime, R. M., Charity, M. J., Harvey, J. T., \& Payne, W. R. (2015). Participation in sport and physical activity: Associations with socio-economic status and geographical remoteness. BMC Public Health, 15(1), 434. https://doi.org/10.1186/s12889-015-1796-0.

[4] Seixas, A. A., Henclewood, D. A., Langford, A. T., McFarlane, S. I., Zizi, F., \& Jean-Louis, G. (2017). Differential and combined effects of physical activity profiles and prohealth behaviors on diabetes prevalence among blacks and whites in the US Population: A novel bayesian belief network machine learning analysis. Journal of Diabetes Research, 1-10. https://doi.org/10.1155/2017/5906034.

[5] Richards, J., Oyeyemi, A., \& Bauman, A. (2017). Physical activity among diverse populations internationally. In Bopp, M. (ed.), Physical Activity in Diverse Populations: Evidence and Practice, 244-272. London: Routledge. https://doi.org/10.4324/9781315561264.

[6] Cappuccio, F. P., Taggart, F. M., Kandala, N. B., Currie, A., Peile, E., Stranges, S., \& Miller, M. A. (2008). Meta-analysis of short sleep duration and obesity in children and adults. Sleep, 31(5), 619-626. doi:10.1093/sleep/31.5.619

[7] Cauter, E. V., \& Knutson, K. L. (2008). Sleep and the epidemic of obesity in children and adults. European Journal of Endocrinology, 159(S1), S59-S66.

[8] Lowry, R., Eaton, D. K., Foti, K., McKnight-Eily, K. L., Perry, G., \& Galuska, D. A. (2012). Association of sleep duration with obesity among US high school students. Journal of Obesity, 2012476914. doi.org/10.1155/2012/476914.

[9] Yeh, C. Y., Chen, I. J., Ku, P. W., \& Chen, C. M. (2015). Modelling the vicious circle between obesity and physical activity in children and adolescents using a bivariate probit model with endogenous regressors. Journal of biosocial science, 47(10, 64-74. doi:10.1017/S0021932013000722.

[10] Carlson, J. A., Crespo, N. C., Sallis, J. F., Patterson, R. E., \& Elder, J. P. (2002). Dietary-related and physical activityrelated predictors of obesity in children: a 2 -year prospective study. Childhood obesity, 8, 110-5. doi:10.1089/chi.2011.0071.

[11] Moreira, P., Santos, S., Padrão, P., Cordeiro, T., Bessa, M., Valente, H., \& ... Moreira, A. (2010). Food patterns according to sociodemographics, physical activity, sleeping and obesity in Portuguese children. International Journal of Environmental Research and Public Health, 7(3), 1121-38. doi:10.3390/ijerph7031121.

[12] Lounsbery, M. F., Holt, K. A., Monnat, S. A., McKenzie, T. L., \& Funk, B. (2014). JROTC as a substitute for PE: Really? Research Quarterly For Exercise \& Sport, 85(3), 414-419.

[13] Centers for Disease Control and Prevention. (2013). National Youth Risk Behavior Surveillance - United States, 2013. Retrieved from https://www.cdc.gov/mmwr/pdf/ss/ss6304.pdf.

[14] Centers for Disease Control and Prevention. (2013). Handbook for conducting Youth Risk Behavior Surveys. Retrieved

from https://samplebox.westat.com/yrbs shp/media/pdf/Sample Bo x_YBRS.pdf.

[15] Hirshkowitz, M., Whiton, K., Albert, S. M., Alessi, C., Bruni, O., DonCarlos, L.; \& ... Ware, J. C.. National Sleep Foundation's updated sleep duration recommendations: Final report. Sleep Health, 1(4), 233-243. https://doi.org/10.1016/j.sleh.2015.10.004.

[16] Levin, S., Lowry, R., Brown, D. R., \& Dietz, W. H. (2003). Physical activity and body mass index among US adolescents: youth risk behavior survey, 1999. Arch Pediatr Adolesc Med, 157(8), 816-820. doi:10.1001/archpedi.157.8.816.

[17] Biddle, S. J. H., Whitehead, S. H. , O’Donovan, T. M., \& Nevill, M. E. (2005). Correlates of participation in physical activity for adolescent girls: a systematic review of recent literature. Journal of Physical Activity and Health, 2(4), 423434.

[18] Knight, J. A. (2012). Physical inactivity: associated diseases and disorders. Annals of Clinical \& Laboratory Science, $42(3), 320-337$.

[19] Wong, G., Kong, A., \& Chan, J. (2009). The metabolic syndrome in children and adolescents. Journal of Diabetes, 1 , A21-A22.

[20] Patrick, K., Norman, G. J., Calfas, K. J., Sallis, J. F., Zabinski, M. F., Rupp, J., \& Cella, J. (2004). Diet, physical activity, and sedentary behaviors as risk factors for overweight in adolescence. Archives of pediatrics \& adolescent medicine, 158(4), 385-390. doi:10.1001/archpedi.158.4.385.

[21] Newby, P. K. (2009). Plant foods and plant-based diets: Protective against childhood obesity? American Journal of Clinical Nutrition, 89(5), 1572S-1587S. https://doi.org/10.3945/ajen.2009.26736G.

[22] Makubuya, T. (2017). Sleep duration and BMI percentile in adolescents: An analysis of the 2013 National Youth Risk Behavioral Survey. MO J Health, Physical Education, Recreation, and Dance, 27, 43-50.

[23] Snell, E. K., Adam, E. K., \& Duncan, G. J. (2007). Sleep and the body mass index and overweight status of children and adolescents. Child Development, 78(1), 309-323.

[24] Reither, E. N., Krueger, P. M., Hale, L., Reiter, E. M., \& Peppard, P. E. (2014). Ethnic variation in the association between sleep and body mass among US adolescents. International Journal of Obesity, 38(7), 944-949.

[25] Chomitz, V. R., McGowan, R. J., Wendel, J. M., Williams, S. A., Cabral, H. J., King, S. E., \& ... Hacker, K. A. (2010). Healthy living Cambridge kids: A community-based participatory effort to promote healthy weight and fitness. Obesity, 18(Supp 1), S45-S53. doi: 10.1038/oby.2009.431.

[26] Economos, C. D., Hyatt, R. R., Goldberg, J. P., Must, A., Naumova, E. N., Collins, J. J., \& Nelson, M. E. (2007). A community intervention reduces BMI z-score in children: Shape Up Somerville first year results. Obesity, 15(5), 13251336.

[27] Sallis, J. F., McKenzie, T. L., Conway, T. L., Elder, J. P, Prochaska, J. J., Brown, M., \& ... Alcaraz, J. E. (2003). Environmental interventions for eating and physical activity: A randomized controlled trial in middle schools. Am J Prev Med., 24(3), 209-217. 
[28] Lubans, D. R., Morgan, P. J., Dewar, D., Collins, C. E., Plotnikoff, R. C., Okely, A. D., \& ... Callister, R. (2010). The Nutrition and Enjoyable Activity for Teen Girls (NEAT girls) randomized controlled trial for adolescent girls from disadvantaged secondary schools: Rationale, study protocol, and baseline results. BMC Public Health, 10(1), 652. doi:10.1186/1471-2458-10-652.

[29] Nader, P. R., Bradley, R. H., Houts, R. M., McRitchie, S. L., \& O'Brien, M. (2008). Moderate-to-vigorous physical activity from ages 9 to 15 years. Journal of the American Medical Association, 300(3), 295-305. doi:10.1001/jama.300.3.295.

[30] De Bourdeaudhuij, I., Van Cauwenberghe, E., Spittaels, H., Oppert, J. M., Rostami, C., Brug, J., \& ... Maes, L. (2011). School-based interventions promoting both physical activity and healthy eating in Europe: A systematic review within the HOPE project. Obes Rev, 12(3), 205-216. doi: 10.1111/j.1467-789X.2009.00711.x

[31] Khambalia, A. Z., Dickinson, S., Hardy, L. L., Gill, T., \& Baur, L. A. (2012). A synthesis of existing systematic reviews and meta-analyses of school-based behavioral interventions for controlling and preventing obesity. Obes Rev, 13(3), 214 233. doi: 10.1111/j.1467-789X.2011.00947.x

[32] Safron, M., Cislak, A., Gaspar, T., \& Luszczynska, A. (2011). Effects of school-based interventions targeting obesity-related behaviors and body weight change: A systematic umbrella review. Behav Med, 37(1), 15-25. https://doi.org/10.1080/08964289.2010.543194

[33] Ash, T., Agaronov, A., Young, T., Aftosmes-Tobio, A., \& Davison, K. K. (2017). Family-based childhood obesity prevention interventions: a systematic review and quantitative content analysis. The International Journal of Behavioral Nutrition and Physical Activity, 14(1), 1-12. https://doi.org/10.1186/s12966-017-0571-2

[34] Driskell, M. M., Dyment, S., Mauriello, L., Castle, P., \& Sherman, K. (2008). Relationships among multiple behaviors for childhood and adolescent obesity prevention. Prev Med., 46(3), 209-15. https://doi.org/10.1016/j.ypmed.2007.07.028 